\title{
Enrichment of heavy elements in the Sextans dwarf Spheroidal Galaxy
}

\author{
Satoshi Honda ${ }^{1}$ \\ Kyoto University, Kwasan Observatory \\ Ohmine-cho Kita Kazan, Yamashina-ku, Kyoto, Japan \\ E-mail: honda@kwasan.kyoto-u.ac.jp \\ Wako Aoki \\ National Astronomical Observatory of Japan \\ 2-21-1 Osawa, Mitaka, Tokyo, Japan \\ E-mail: aoki.wako@nao.ac.jp
}

\section{Nobuo Arimoto}

National Astronomical Observatory of Japan

2-21-1 Osawa, Mitaka, Tokyo, Japan

E-mail: arimoto.nenao.ac.jp

\section{Kozo Sadakane}

Osaka Kyoiku University

4-698-1 Asahigaoka, Kashiwara-shi, Osaka, Japan

E-mail: sadakane@osaka-kyoiku.ac.jp

\begin{abstract}
We determined chemical abundances of extremely metal-poor (EMP) stars in the Sextans dwarf galaxy. The heavy element Ba is deficient in EMP stars in dwarf galaxies in general, while some fraction of EMP stars in the Milky Way halo show large excesses of this element. Sextans, however, we identified one object (S15-19) that shows exceptionally high Ba abundance $([\mathrm{Ba} / \mathrm{Fe}] \sim+0.4)$, which is as high as the values found in r-process enhanced stars in the Milky Way (so-called r-II stars). We recently conducted high resolution spectroscopy for this object to determine the origin of the heavy elements in this object. The low upper-limit of the Eu abundance indicates that the $\mathrm{Ba}$ of this object is not explained by the r-process, but s-process at least partially contributed to the enrichment of heavy elements. The carbon-excess and the radial velocity variation found for this object suggest that the Ba excess is attributed to the s-process in an AGB star and the mass transfer across the binary system.
\end{abstract}

11th Symposium on Nuclei in the Cosmos - NIC XI

Heidelberg, Germany

July 19-232010

$1 \quad$ Speaker 


\section{Introduction}

A few tens of dwarf galaxies have been found around our Milky Way (MW) Galaxy. They are believed to play key roles in understanding the formation processes of the MW halo structure (c.f.,[1]). The chemical composition of individual stars of dwarf galaxies can be derived by high-resolution spectroscopy with $8-10 \mathrm{~m}$ telescopes (e.g.,[2]). Shetrone et al. [3] investigated the abundances of several stars in Draco, Sextans, and Ursa Minor using Keck/HIRES. They found that the abundances of alpha elements of a large fraction of stars in dwarf galaxies are lower than that of Milky Way. However, since only a few extremely metalpoor (EMP) stars in dwarf galaxies are found, the behavior of chemical abundances in the very metal-poor region $([\mathrm{Fe} / \mathrm{H}]<-2.5)$ were unclear.

Helmi et al. [4] found EMP candidate stars in dwarf galaxies in the DART (Dwarf Abundances and Radial velocities Team [5]) program with VLT/FLAMES. Recently, chemical compositions of candidates of EMP stars in the Sextans dwarf galaxies found by Helmi et al. were studied in detail with Subaru/HDS by Aoki et al. [6]. They found that the $[\mathrm{Mg} / \mathrm{Fe}]$ values of most stars are significantly lower than those found in MW halo stars, as seen in other dwarf galaxy stars with higher metallicity.

Another interesting feature found by them is that the abundances of $\mathrm{Ba}$ in Sextans stars show large scatter as in MW halo. Among them one EMP star (S15-19) shows an exceptionally large excess of $\mathrm{Ba}$ (Figure 1).

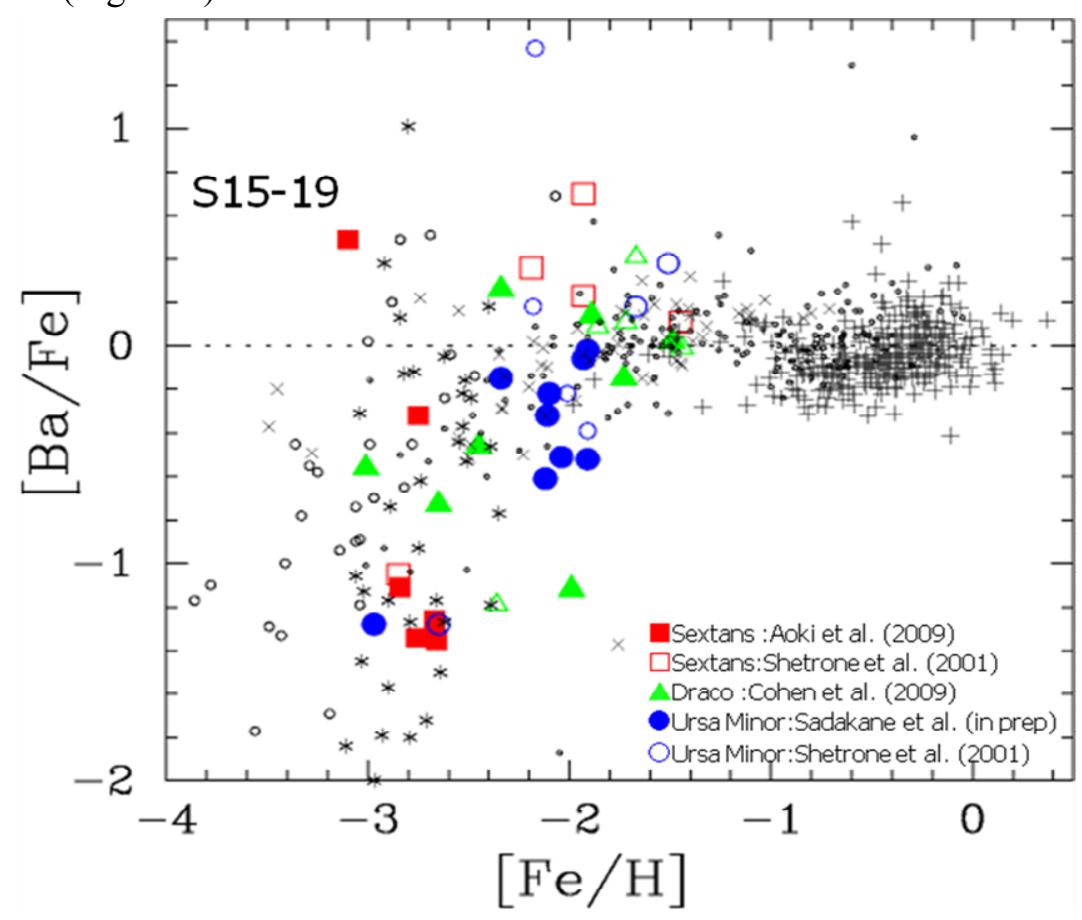

Figure 1. Ba abundance ratio as a function of iron abundance. The abundances of MW halo stars are shown by black small symbols.

The EMP stars of the MW halo show large dispersion in the abundance of neutron-capture elements including Ba (e.g., [7]). The abundance patterns of EMP stars show overabundances of 
neutron-capture elements are usually similar to the solar-system r-process pattern (e.g.,[8]). This is interpreted as a result of short time-scale of r-process events compared to the enrichment of sprocess elements by evolved intermediate-mass stars (AGB stars), although the astrophysical site of the r-process is still not well understood. Such a scenario of heavy element enrichment can be examined by the measurements of heavy elements in dwarf galaxies around the MW for which individual stars can be studied based on high resolution spectroscopy (e.g., [10,11]).

Recently, EMP stars in ultra-faint dwarf galaxies, which are newly found dwarf galaxies with extremely low luminosity, have been studied (e.g. [12]), and their abundances of neutroncapture elements are found to be very low. Hence, Sextans S15-19 is a unique object having excesses of heavy neutron-capture elements among EMP stars in dwarf galaxies known at present. However, since only one heavy neutron capture element $(\mathrm{Ba})$ was detected by Aoki et al. [6], the origin of the excess was unclear. Indeed, some MW field EMP stars show excesses of heavy elements that can be attributed to s-process. Such stars usually show large excesses of carbon, and their origin should be AGB stars. We here report the result of our abundance study for S15-19 to distinguish the origin of its Ba excess.

\section{High dispersion spectroscopy and abundance analysis of S15-19}

High resolution spectroscopy was carried out for S15-19 in 2010 Feb. 9 using Subaru/HDS [13]. The spectrum covers $3760-5490 \AA$ with a resolving power of 40,000 and $2 \times 2$ on-chip binning. This spectral range includes atomic lines of $\mathrm{Eu}, \mathrm{Sr}$, and $\mathrm{CH}$ molecular bands. The long exposure (8hr) achieved a signal-to-noise $(\mathrm{S} / \mathrm{N})$ ratio of 50 (per resolution element) at $4500 \AA$. Equivalent widths were measured by fitting gaussian profiles. Measurement of radial velocity was made for selected clean iron lines used in the equivalent width measurements. Derived radial velocity was $223.60 \pm 0.23 \mathrm{~km} / \mathrm{s}$. This value is significantly different from that obtained by the previous study [6] $(226.05 \pm 0.11 \mathrm{~km} / \mathrm{s})$, suggesting that $\mathrm{S} 15-19$ belongs to a binary system.

Chemical abundance analyses were performed using the analysis program SPTOOL developed by Y. Takeda (private communication), based on Kurucz's ATLAS9/WIDTH9 [14]. We compute the spectra under the assumption of local thermodynamic equilibrium (LTE). We adopted the model atmosphere parameters (effective temperature, gravity, microturbulent velocity, and metallicity) derived by previous study [6]. The model atmosphere used for abundance analysis is $T_{\text {eff }}=4600 \mathrm{~K}, \log =1.2, \mathrm{vt}=2.6$, and $[\mathrm{Fe} / \mathrm{H}]=-3.1$. We derive the iron abundance of this star $([\mathrm{Fe} / \mathrm{H}]=-3.05)$ adopting the average of the results from $\mathrm{Fe}$ I and $\mathrm{Fe}$ II lines.

We confirm the metallicity $([\mathrm{Fe} / \mathrm{H}])$ of this star is lower than -3 and that $\mathrm{Ba}$ abundance is high, $[\mathrm{Ba} / \mathrm{Fe}] \sim+0.4$. However, neutron-capture elements heavier than $\mathrm{Ba}$ have not been detected. We derived upper limits for some neutron-capture elements, including Eu. The upper limit of the Eu abundance $([\mathrm{Eu} / \mathrm{Fe}]<+0.9)$ is lower than the value expected assuming that the neutron-capture elements of this star was produced by r-process only (Figure 2). The ratio $[\mathrm{Eu} / \mathrm{Ba}]$, which reflects the ratio of r-process to s-process abundances, is lower than +0.5 , which is lower than the value expected for r-process $(+0.8$ [15]). Among lighter neutron-capture elements, we detect the $\mathrm{Sr} 4077 \AA$ line and derived the abundance $([\mathrm{Sr} / \mathrm{Fe}]=-1.4)$. The $\mathrm{Sr}$ 
abundance is also lower than the value expected from $\mathrm{Ba}$ abundance and the r-process abundance pattern.

Another support for the s-process contribution is found in the carbon abundance of this object. The carbon abundance is determined by the spectrum synthesis of the $\mathrm{CH}$ G-band at $4324 \AA$. The result clearly shows that this star is carbon-enhanced $([\mathrm{C} / \mathrm{Fe}]=+1.0)$, which is a signature of contribution by AGB nucleosynthesis.

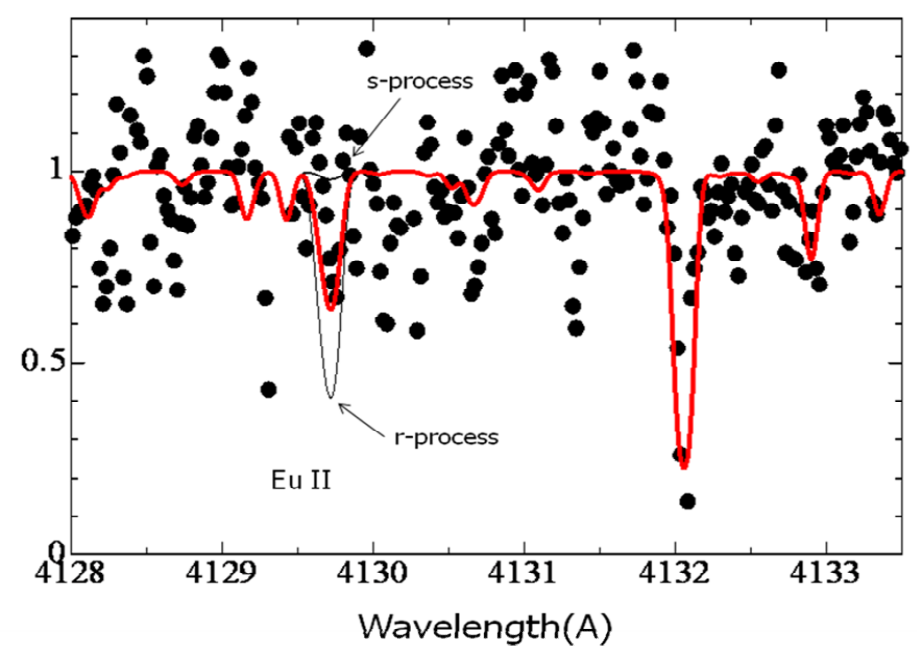

Figure 2. Observed and synthetic spectra of the Eu II (4129A) region for S15-19.

The synthetic spectra are shown for three Eu abundances: top is that for the s-process case, while the bottom is the r-process one, which are calculated adopting $\mathrm{Ba}$ abundance. The middle is the case of our estimated upper limit.

\section{Summary and concluding remarks}

We derived the abundance of neutron-capture elements in the EMP star S15-19 in the Sextans dwarf galaxy. We confirm that S15-19 is a Ba enhanced EMP star. The upper limit of the Eu abundance shows that neutron-capture elements of this star are not originated only from r-process. In addition, we found that this star is a carbon rich star belonging to a binary system. This result indicates that the Ba excess of this star is a result of s-process in an AGB star and the mass transfer across a binary system.

S15-19 is a unique object that shows large overabundance of Ba among EMP stars in dwarf galaxies. Since the origin of Ba of this object turned out to be s-process, no r-process enhanced EMP star is known at present in dwarf galaxies yet (c.f., [12]). This suggests that dwarf galaxies may have chemical evolution different from MW. However, the current sample is still insufficient to derive definitive conclusion, and further abundance measurements for EMP stars in dwarf galaxies are desirable to clarify the chemical evolutions of dwarf galaxy and nucleosynthesis of r-process.

\section{References}

[1] Frebel in proceedings of Nuclei in the Cosmos XI 
[2] Tolstoy, E., Hill, V., \& Tosi, M. ARA\&A, 47, 371 (2009)

[3] Shetrone, M., Venn, K. A., Tolstoy, E., et al. AJ, 125, 684 (2003)

[4] Helmi, A., Irwin, M. J., Tolstoy, E., et al. ApJ, 651, L121 (2006)

[5] Tolstoy, E., Irwin, M.J., \& Helmi, A., et al. ApJ, 617, L119 (2004)

[6] Aoki, W. et al. $A \& A$ 502, 569 (2009)

[7] Honda, S., Aoki, W., Kajino, T., et al. ApJ, 607, 474 (2004)

[8] Sneden, C., Cowan, J. J., Lawler, J. E., et al. ApJ, 591, 936 (2003)

[9] Beers, T. C., \& Christlieb, N. $A R A \& A$, 43, 531 (2005)

[10] Argast, D., Samland, M., Thielemann, F.-K. et al. $A \& A, 416,997$ (2004)

[11] Cescutti, G., François, P., Matteucci, F. et al. $A \& A, 448,557$ (2006)

[12] Frebel, A., Simon, J. D., Geha, M., \& Willman, B. ApJ, 708, 560 (2010)

[13] Noguchi, K. et al. PASJ, 54. 855 (2002)

[14] Kurucz, R. ATLAS9 Stellar Atmosphere Programs and $2 \mathrm{~km} \mathrm{s-1}$ grid. Kurucz CD-ROM No. 13, Cambridge, Mass., Smithsonian Astrophysical Observatory (1993)

[15] Burris, D. L., Pilachowski, C. A., Armandroff, T. A., et al. ApJ, 544, 302 (2000) 